\title{
Classificação das Universidades Federais Brasileiras com base nos indicadores de gestão propostos pelo Tribunal de Contas da União - TCU
}

\author{
Alexandre Severino Pereira - alexandre.severinop@gmail.com ${ }^{1}$ \\ Luciana Fernanda Puppin Pereira - lucianaufes@yahoo.com.br ${ }^{2}$ \\ Mariana Recla de Tognini - marianarecla.d@gmail.com ${ }^{3}$ \\ Maria Auxiliadora de Carvalho Corassa - corassa@terra.com.br ${ }^{4}$ \\ Teresa Cristina Janes Carneiro - carneiro.teresa@gmail.com ${ }^{4}$
}

Resumo - O objetivo do estudo foi classificar as universidades federais brasileiras de acordo com os indicadores de gestão propostos pelo Tribunal de Contas da União - TCU e verificar o potencial desses indicadores para classificar as instituições de ensino. Os dados foram obtidos de 59 relatórios de gestão referentes ao exercício de 2012. Foram utilizadas técnicas multivariadas de análise de agrupamento e análise discriminante que dividiu as universidades em 3 grupos. O Grupo 1 foi composto por 28 universidades com maior número de alunos, professores e funcionários e maiores valores de desempenho discente e custo corrente bruto. O Grupo 2 foi caracterizado pelas 17 universidades com pequena quantidade de alunos da graduação em relação ao tamanho da estrutura existente. O Grupo 3 reuniu seis universidades com o pior desempenho discente, os menores níveis de qualificação dos professores e de investimento por aluno. O modelo discriminante gerado comprovou a diferença estatística entre os grupos e foi capaz de classificar corretamente $100 \%$ das observações.

Palavras-chave: Universidades federais, Indicadores de gestão, Avaliação do ensino superior, Análise de agrupamentos, Análise discriminante.

\section{Brazilian Federal Universities Under TCU Management Indicators Proposals: A Classification Scheme}

\begin{abstract}
The objective of this study was ranking Brazilian federal universities, according to management indicators, proposed by the National Audit Court (Tribunal de Contas da União - TCU) and check the potential use of these indicators to rank educational institutions. Data was collected from 59 management reports of the financial year of 2012. Multivariate techniques were used, with cluster analysis and discriminant analysis, so that universities were divided into 3 groups. Group 1 comprises 28 universities, with the biggest number of students, professors and staff, highest level of student performance and highest level of current gross cost. Group 2 comprises 17 universities with the smallest amount of undergraduate students, when considering the existing structure. 3 comprises 6 universities with the worst student performance, the lowest level of education for professors and the lowest level of investment per student. The discriminant model created showed statistical differences between groups and it was able to rank appropriately $100 \%$ of the observations.
\end{abstract}

Keywords: federal universities, management indicators, higher education assessment, cluster analysis, discriminant analysis.

Data de Submissão: 29/04/2020

Data de Aceitação:29/04/2020

${ }^{\text {I }}$ Universidade Federal do Espírito Santo - Mestrado Profissional em Gestão Pública e do Programa de Pós-

Graduação em Administração 


\section{INTRODUÇÃO}

A avaliação institucional da educação superior é um tema complexo (DIAS SOBRINHO, 2002). Essa complexidade da avaliação advém da estrutura organizacional das universidades, geralmente formal e burocrática, que engessa o gerenciamento; e da estrutura das relações de poder entre os atores (professores, técnicos, estudantes e sociedade) (GUERRA; BARBOSA, 2006)

Em função disso, diferentes metodologias de avaliação de resultados têm sido utilizadas nos últimos anos, tais como: Programa da Avaliação Institucional das Universidades Brasileiras, Exame Nacional de Cursos, Análise das Condições de Ensino, Proposta de Avaliação do Conselho de Reitores das Universidades Brasileiras e Sistema Nacional de Avaliação da Educação Superior (SINAES) (GUERRA; BARBOSA; 2006).

Dentro desse conjunto de metodologias, desde 2002 as Instituições Federais de Ensino Superior (IFES) têm passado por um processo de avaliação contínua do seu desempenho operacional, cujo procedimento foi definido pela Decisão $n^{0}$ 408/2002 do Tribunal de Contas da União (TCU). O TCU determinou a inclusão de nove indicadores de desempenho nos relatórios de gestão das IFES, com o intuito de construir uma série histórica da evolução de aspectos gerenciais relevantes, servindo de instrumento de acompanhamento do desempenho e aprimoramento da gestão das instituições (SANTOS; CASTANEDA; BARBOSA; 20II).

Os indicadores propostos medem aspectos do desempenho e tem por objetivo permitir comparações com desempenhos anteriores e com metas estabelecidas. Adicionalmente, podem permitir comparação de desempenho das instituições de ensino similares.

Sendo assim, o objetivo deste trabalho é verificar o potencial dos indicadores de desempenho propostos pelo TCU de atuarem como classificadores das instituições de ensino superior e realizar comparações entre instituições de um mesmo grupo e de grupos diferentes.

Caso se mostrem adequados como classificadores das instituições de ensino superior, esses indicadores poderão ser utilizados como instrumentos de melhoria de gestão não apenas pela comparação com resultados passados e metas pretendidas, mas também pela comparação com outras instituições com características similares e melhores desempenhos.

O artigo aborda, além dessas considerações iniciais, o Referencial Teórico sobre avaliação do ensino superior e indicadores de gestão das IFES; descreve na Metodologia os procedimentos de coleta e análise dos dados e apresenta os resultados obtidos e as conclusões do estudo.

\section{AVALIAÇÃO DO ENSINO SUPERIOR}

Os anos 1990 foram marcados por reformas administrativas no setor público que implementaram políticas de autoavaliação institucional. A educação superior foi incluída nessas mudanças. O Governo Federal pretendia tornar o ambiente universitário mais eficiente e produtivo 
visando a redução de gastos, aumento das pesquisas, ampliação de pós-graduação, capacitação e valorização dos profissionais da educação (FREIRE; CRISÓSTOMO; CASTRO, 2008). Um ponto relevante das reformas foi a prestação de contas dos atos administrativos à sociedade, conforme colocado por Santos, Castaneda e Barbosa (20II, p.2):

\footnotetext{
As Instituições Federais de Ensino Superior (IFES), enquanto órgãos integrantes da estrutura federal têm por obrigação legal tornarem públicas as suas realizações e a aplicação dos seus recursos através de relatórios de gestão, divulgando-os entre os órgãos competentes e, principalmente, entre a comunidade universitária e a sociedade na qual está inserida.
}

A avaliação das instituições federais de ensino superior é conduzida por duas vertentes: o desempenho da gestão e a qualidade acadêmica. Essas avaliações são conduzidas de um lado, pelo Tribunal de Contas da União (TCU), que através da decisão ${ }^{\circ}$ 408/2002 exigiu que as IFES agregassem em seus relatórios de gestão nove indicadores de desempenho (BRASIL, 2002). Por outro lado, pelo Ministério da Educação (MEC), que por meio da Lei no ${ }^{\circ}$ o.86i/2004, instituiu o Sistema Nacional de Avaliação da Educação Superior (SINAES) para nortear a avaliação da qualidade do ensino superior em três eixos: avaliação das instituições, avaliação dos cursos e avaliação de desempenho dos alunos (BRASIL, 2004).

O processo de avaliação de instituições de ensino, como colocado por Freire, Crisóstomo e Castro (2008) [...] "serve de instrumento de autoconhecimento, de tomada de decisão e de aperfeiçoamento permanente". O que ressalta a importância do uso de indicadores para auxiliar no processo de tomada de decisão, alocação de recursos e o aprimoramento das atividades.

\subsection{Indicadores de Gestão}

Os indicadores de gestão propostos pelo TCU surgem com o intuito de compor um diagnóstico da gestão do ensino superior brasileiro. Consta da decisão no 408/2002 que os indicadores foram estabelecidos a partir de auditorias realizadas em instituições federais de ensino superior, inicialmente na Universidade de Brasília (UNB) e posteriormente estendidas à Universidade do Amazonas (UFAM), Universidade Federal de Goiás (UFG), Universidade Federal de Pernambuco (UFPE), Universidade Federal do Rio de Janeiro (UFRJ) e Universidade Federal do Rio Grande do Sul (UFRGS) (SILVA; MORGAN; COSTA, 2004).

Dessa forma, segundo Freire, Crisóstomo e Castro (2007) e Brasil (2002) foram propostos os seguintes indicadores de gestão:

- Custo Corrente / Aluno Equivalente: são despesas correntes de todas as unidades gestoras da instituição sem considerar despesas com sentenças judiciais, aposentadorias e pensões, servidores afastados ou cedidos e despesas com hospitais e maternidades universitários. Aluno equivalente designa o número de alunos equivalentes da graduação, número de alunos tempo integral de pós-graduação e residência médica. Inclui todos os cursos de caráter permanente e exclui aqueles que são autofinanciáveis por meio de parcerias com entes públicos ou privados. 
- Aluno tempo integral / professor equivalente: relaciona o número de alunos em tempo integral com o número de professores equivalentes com ou sem dedicação exclusiva. O cálculo do professor equivalente considera os pesos: 0,5 para professores com carga horária de 20 horas por semana e peso I para professores com dedicação exclusiva ou 40 horas de carga horária semanal.

- Aluno tempo integral / funcionário equivalente: relaciona o número de alunos tempo integral com a força de trabalho administrativo da instituição. O cálculo do funcionário equivalente considera os pesos: 0,5 para funcionários com carga horária de 20 horas semanais, 0,75 para 30 horas semanais e pesoI para 40 horas semanais.

- Funcionário equivalente / professor equivalente: relaciona as forças de trabalho da instituição (professor e funcionários).

- Grau de participação estudantil: indica o número de alunos da graduação dedicados em tempo integral ao curso. Mede o grau de utilização, pelos discentes, da capacidade instalada da IFES e a velocidade de integralização curricular.

- Grau de envolvimento com pós-graduação: mostra a profundidade do engajamento discente com a pós-graduação stricto sensu. É a razão entre o total de alunos matriculados em programas de mestrado e doutorado e o total de alunos matriculados na instituição.

- Conceito CAPES/MEC para pós-graduação: considera a relação entre a média aritmética das notas de avaliação da Capes de todos os cursos de mestrado e doutorado (não considera mestrados profissionais) e o número de programas de pós-graduação.

-Índice de qualificação do corpo docente: indica o grau de qualificação dos professores, de acordo com as seguintes ponderações: professor doutor - peso 5, professor mestre - peso 3, professor especialista - peso 2, professor graduado - peso I. Esse resultado é dividido pelo número total de professores.

- Taxa de sucesso na graduação: relaciona o número de concluintes (que concluíram todos os créditos) com o total de alunos ingressantes.

Esses indicadores propostos são informados pelas instituições federais de ensino superior (IFES), anualmente, em seus relatórios de gestão enviados ao TCU.

\section{METODOLOGIA}

Este artigo é um estudo descritivo de abordagem quantitativa. Visa classificar as IFES segundo seus indicadores de gestão e apresentar uma descrição do desempenho dos grupos identificados. A abordagem quantitativa advém da utilização de recursos de estatística descritiva e técnicas de análises multivariadas, realizadas com auxílio dos softwares Statistical Package for the Social Sciences versão 20 (SPSS 20) e Microsoft Office Excel 2007.

\subsection{Escolha dos indicadores}

A primeira etapa do trabalho foi a escolha dos indicadores a serem utilizados. Para escolha dos indicadores foi considerado a possibilidade de acesso aos dados, a padronização das formas de cálculo e a abrangência das IFES. Assim, foram adotados nove indicadores de gestão constante da Decisão $n^{0}$ 408/2002-TCU que todas IFES devem apresentar em seus Relatórios 
de Gestão (prestação de contas anual). Foram excluídos os indicadores relativos aos Hospitais Universitários considerando que nem todas as universidades possuem hospital escola.

Os dados foram coletados dos Relatórios de Gestão das IFES relativos ao ano de exercício de 20I2, tendo em vista que parte dos documentos referentes a 2013 ainda não estavam disponíveis no sítio de consulta pública disponibilizado pelo TCU no período de realização do estudo. Foram identificados e obtidos 59 relatórios de gestão das universidades federais.

\subsection{Procedimentos de análise dos dados}

As análises foram iniciadas por uma etapa descritiva. Em seguida foi aplicada a técnica de Análise de Agrupamentos. Os agrupamentos gerados foram então submetidos à validação pela Análise Discriminante. Os procedimentos de cada etapa são descritos a seguir.

A primeira parte da análise dos dados teve como objetivo descrever as características gerais dos indicadores através do uso de medidas estatísticas de tendência central e dispersão. Os gráficos boxplot (gráfico de caixa) foram utilizados para avaliar a distribuição empírica dos dados e identificar os valores extremos constantes no conjunto de dados. Também foram calculados os coeficientes de correlação para identificar casos de alta correlação entre os indicadores, o que pode afetar os resultados produzidos pelas técnicas de análise de agrupamento e discriminante.

A segunda parte da análise foi a análise de agrupamentos (também conhecida como análise de cluster ou de conglomerados), utilizada com o objetivo de classificar as IFES com base em seus indicadores de gestão. Espera-se que as instituições com desempenho semelhante sejam reunidas em grupos de elevada homogeneidade interna, assim como os agrupamentos apresentem elevada heterogeneidade externa entre si, conforme apontado por Hair et al (2009).

Para utilização dessa técnica foram desconsiderados casos de universidades com dados ausentes e valores extremos, além de indicadores com alta correlação entre si. Esse procedimento também foi utilizado na próxima etapa.

A terceira parte consistiu na análise discriminante, uma técnica utilizada para estimar a relação entre uma variável dependente não métrica (categórica) e um conjunto de variáveis independentes métricas (HAIR et al, 2009). Segundo Maroco (2003), essa técnica visa: (a) identificar as variáveis que melhor discriminam dois ou mais grupos estruturalmente diferentes e mutuamente exclusivos; (b) utilizar essas variáveis para criar uma função discriminante que represente de forma parcimoniosa as diferenças entre os grupos; e (c) utilizar essa função discriminante para classificar a priori novos indivíduos nos grupos.

Nesse estudo, a técnica foi aplicada como procedimento confirmatório da análise de agrupamentos, conforme indicado por Fávero et al (2009), visando confirmar a diferença entre os grupos gerados na etapa anterior e apontar as variáveis que mais bem discriminam os grupos. A validade das soluções geradas foi verificada com base no teste Box’M, na significância da estatística Lambda de Wilks e das funções discriminantes e nos resultados da Tabela de Classificação. 


\section{RMP Revista dos Mestrados

\section{RESULTADOS E DISCUSSÃO}

\subsection{Análise Descritiva e Tratamento dos Dados}

Figura I.

Os valores mínimo, máximo, médio e desvio padrão dos indicadores de gestão das universidades federais são apresentados na Tabela I e

Tabela I - Estatísticas descritivas dos Indicadores de Gestão das Universidades Federais - 20 I2

\begin{tabular}{|c|c|c|c|c|c|c|}
\hline Indicador de gestão [*] & $\mathrm{N}$ & Média & Desvio padrão & Mínimo & Máximo & Mediana \\
\hline Custo Corrente / Aluno Equivalente (R\$) [a] & 59 & $15.552,54$ & $4.912,55$ & $8.237,03$ & $35.317,84$ & $14.359,32$ \\
\hline Aluno Tempo Integral / Professor Equivalente [b] & 59 & 11,13 & 2,96 & 3,65 & 16,46 & 11,42 \\
\hline Aluno Tempo Integral / Funcionário Equivalente & 59 & 7,97 & 2,84 & 2,56 & 16,68 & 8,10 \\
\hline $\begin{array}{l}\text { Funcionário Equivalente / Professor Equivalente } \\
\text { [d] }\end{array}$ & 59 & 1,50 & 0,42 & 0,50 & 3,05 & 1,43 \\
\hline Grau de Participação Estudantil [e] & 59 & 0,69 & 0,17 & 0,21 & 1,31 & 0,70 \\
\hline $\begin{array}{l}\text { Grau de Envolvimento Discente com Pós-Gradu- } \\
\text { acão [f] }\end{array}$ & 56 & 0,10 & 0,07 & 0,01 & 0,31 & 0,10 \\
\hline Conceito Capes/MEC para a Pós-Graduação [g] & 57 & 3,70 & 0,66 & 1,06 & 5,11 & 3,67 \\
\hline Índice de Qualificação do Corpo Docente [h] & 59 & 4,11 & 0,39 & 3,27 & 5,00 & 4,11 \\
\hline Taxa de Sucesso na Graduação (\%) [i] & 56 & 54,81 & 18,51 & 13,92 & 116,30 & 54,39 \\
\hline
\end{tabular}

[*] Indicação do boxplot correspondente apresentado na Figura I.

O custo corrente / aluno equivalente das universidades federais brasileiras, em média, foi de R\$ 15.552,54. No entanto, chama a atenção o valor de R \$ 35.317,84 na UNIPAMPA, configurando um valor extremo facilmente visualizado no boxplot (a) da Figura I. A diferença observada entre o valor médio ( $\mathrm{R}$ \$ 15.552,54) e o mediano (R \$ 14.359,32) revelou influência dos valores extremos sobre a média. As universidades brasileiras trabalharam com II,I3 Alunos Tempo Integral / Professor Equivalente (ATIPE) e 7,97 Alunos Tempo Integral / Funcionário Equivalente (ATIFE), sem casos de valores extremos em ambos indicadores.

O indicador Funcionário Equivalente / Professor Equivalente (FEPE) apresentou valor médio de I,5, indicando que a força de trabalho ligada às atividades da área meio é 50\% maior do que a força de trabalho alocada à área fim. Para esse indicador, um valor extremo de 3,05 foi observado na UFV, conforme se verifica no boxplot (d) da Figura I.

19

Pereira, A; Pereira, L; Tognini, M; Corassa, M; Carneiro, T - Classificação das Universidades Federais Brasileiras com base nos indicadores de gestão propostos pelo Tribunal de Contas da União - TCU 


\section{RMP Revista dos Mestrados \\ Profissionais - RMP}

ISSN - 2317 - 0115

HTTPS://PERIODICOS.UFPE.BR/REVISTAS/RMP

v. 6 , n. 2. (2017)

Recife - PE - UFPE / CCSA - MGP

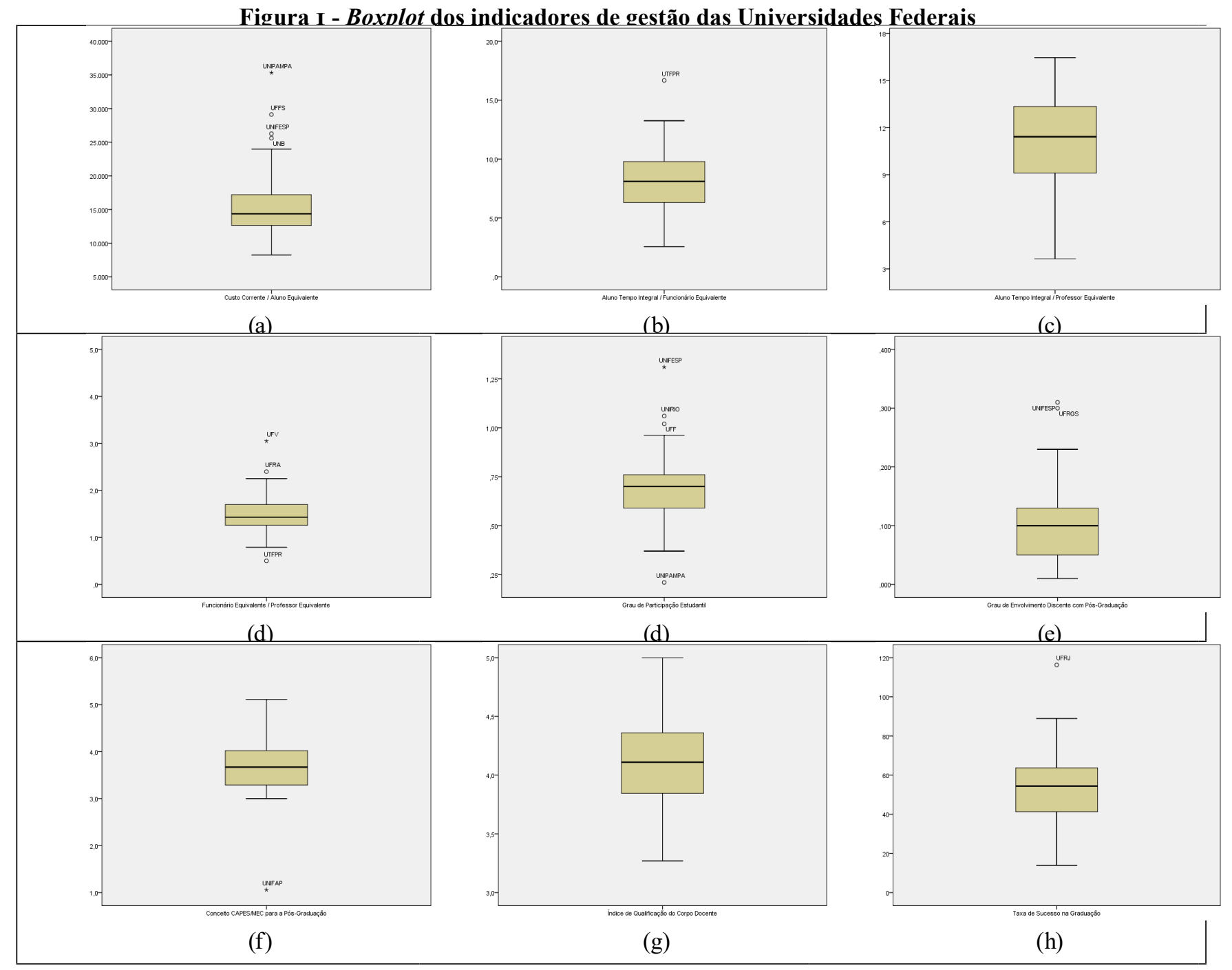

Fonte: Dados da pesquisa, 20I4.

20

Pereira, A; Pereira, L; Tognini, M; Corassa, M; Carneiro, T - Classificação das Universidades Federais Brasileiras com base nos indicadores de gestão propostos pelo Tribunal de Contas da União - TCU 
No indicador Grau de Participação Estudantil (GPE) verificou-se que em média 69\% dos alunos da graduação dedicam-se integralmente ao curso. Nesse indicador foram identificados quatro casos de valores extremos (UNIFESP, UNIRIO, UFF e UNIPAMPA), dos quais a UNIFESP atingiu uma faixa extremamente discrepante.

O indicador Grau de Envolvimento Discente com a Pós-Graduação (GEDPG) aponta que, em média, o quantitativo de alunos dos cursos de pós-graduação stricto sensu equivale a I0\% do total de alunos dos cursos de graduação e pós-graduação. Destaca-se o fato das universidades UNIFESP e UFRGS atingirem um nível de 30\% para esse indicador.

O indicador Conceito Capes/MEC para Pós-Graduação (CCPG) obteve um valor médio de 3,70 pontos. Para esse indicador, destaca-se que duas universidades alcançaram valores superiores a 5,00 pontos, a UFRGS $(5, \mathrm{II})$ e a UFMG $(5,07)$, enquanto a UNIFAP relatou um valor extremamente baixo $(\mathrm{I}, 06)$, o único abaixo de 3,00 pontos.

O Índice de Qualificação do Corpo Docente obteve um valor médio de 4,II, revelando que o corpo docente das IFES analisadas é composto em sua maioria por professores com titulação de doutorado. A média da Taxa de Sucesso na Graduação das IFES brasileiras foi de $54,8 \mathrm{I} \%$, indicando que pouco mais da metade dos alunos dos cursos de graduação das universidades federais concluem o curso no tempo esperado conforme fixado pela SESu/MEC. Para esse indicador, o valor relatado pela UFRJ (II6,3\%) foi considerado discrepante, tendo em vista ainda que superou o valor máximo para o indicador em condições normais.

Na sequência foram analisados os níveis de correlação entre os indicadores de gestão das IFES. Os coeficientes de correlação de Pearson, o p-valor (significância) e o número de observações utilizado na análise são apresentados Tabela 2. Os dados mostram que, a um nível de significância de $5 \%$, existe correlação linear entre diversos indicadores analisados.

O Custo Corrente / Aluno Equivalente possui correlação negativa com os indicadores Aluno Tempo Integral / Professor Equivalente, Aluno Tempo Integral / Funcionário Equivalente e Grau de Participação Estudantil e possui correlação positiva com o Índice de Qualificação do Corpo Docente. Já a Taxa de Sucesso da Graduação possui correlação positiva com Aluno Tempo Integral / Professor Equivalente, Aluno Tempo Integral / Funcionário Equivalente, Grau de Envolvimento discente com a Pós-graduação e Conceito CAPES/MEC para a Pós-Graduação.

Desse resultado destacam-se as altas correlações $(r>0,700)$ identificadas entre os indicadores Conceito Capes/MEC para a Pós-Graduação e Índice de Qualificação do Corpo Docente com o Grau de Envolvimento Discente com a Pós-Graduação. 


\section{RMP \\ Profissionais - RMP}

TPS://PERIODICOS.UFPE.BR/REVISTAS/RMP
ISSN - 2317 - 0115

v. 6 , n. 2. (2017)

Recife - PE - UFPE / CCSA - MGP

Tabela 2 - Correlação entre Indicadores de Gestão das Universidades Federais

\begin{tabular}{|c|c|c|c|c|c|c|c|c|c|c|}
\hline Indicador & & CCAE & ATIPE & ATIFE & FEPE & GPE & GEDPG & $\mathrm{CCPG}$ & IQCD & TSG \\
\hline \multirow{2}{*}{ CCAE } & $\mathrm{r}$ & 1 & $-0,517$ & $-0,574$ & 0,256 & $-0,314$ & 0,094 & $-0,069$ & 0,349 & $-0,221$ \\
\hline & p-valor & & 0,000 & $\mathbf{0 , 0 0 0}$ & 0,050 & 0,016 & 0,491 & 0,610 & 0,007 & 0,102 \\
\hline \multirow{2}{*}{ ATIPE } & $\mathrm{r}$ & $-0,517$ & 1 & 0,551 & 0,138 & 0,663 & 0,601 & 0,463 & 0,139 & 0,413 \\
\hline & p-valor & 0,000 & & 0,000 & 0,299 & 0,000 & 0,000 & 0,000 & 0,294 & 0,002 \\
\hline \multirow{2}{*}{ ATIFE } & $\mathrm{r}$ & $-0,574$ & 0,551 & 1 & $-0,677$ & 0,379 & 0,119 & 0,007 & $-0,230$ & 0,289 \\
\hline & p-valor & 0,000 & 0,000 & & 0,000 & 0,003 & 0,380 & 0,960 & 0,080 & 0,031 \\
\hline \multirow{2}{*}{ FEPE } & $\mathrm{r}$ & 0,256 & 0,138 & $-0,677$ & 1 & 0,073 & 0,308 & 0,365 & 0,377 & 0,005 \\
\hline & p-valor & 0,050 & 0,299 & 0,000 & & 0,582 & 0,021 & 0,005 & 0,003 & 0,969 \\
\hline \multirow{2}{*}{ GPE } & $\mathrm{r}$ & $-0,314$ & 0,663 & 0,379 & 0,073 & 1 & 0,565 & 0,342 & 0,258 & 0,232 \\
\hline & $\mathrm{p}$-valor & 0,016 & 0,000 & 0,003 & 0,582 & & 0,000 & 0,009 & 0,048 & 0,085 \\
\hline \multirow{2}{*}{ GEDPG } & $\mathrm{r}$ & 0,094 & 0,601 & 0,119 & 0,308 & 0,565 & 1 & 0,749 & 0,706 & 0,306 \\
\hline & p-valor & 0,491 & 0,000 & 0,380 & 0,021 & 0,000 & & 0,000 & 0,000 & 0,022 \\
\hline \multirow{2}{*}{ CCPG } & $\mathrm{r}$ & $-0,069$ & 0,463 & 0,007 & 0,365 & 0,342 & 0,749 & 1 & 0,642 & 0,317 \\
\hline & p-valor & 0,610 & 0,000 & 0,960 & 0,005 & 0,009 & $\mathbf{0 , 0 0 0}$ & & 0,000 & 0,017 \\
\hline \multirow{2}{*}{ IQCD } & $\mathrm{r}$ & 0,349 & 0,139 & $-0,230$ & 0,377 & 0,258 & 0,706 & 0,642 & 1 & 0,143 \\
\hline & p-valor & 0,007 & 0,294 & 0,080 & 0,003 & 0,048 & 0,000 & 0,000 & & 0,292 \\
\hline \multirow{2}{*}{ TSG } & $\mathrm{r}$ & $-0,221$ & 0,413 & 0,289 & 0,005 & 0,232 & 0,306 & 0,317 & 0,143 & 1 \\
\hline & $\mathrm{p}$-valor & 0,102 & 0,002 & $\mathbf{0 , 0 3 1}$ & 0,969 & 0,085 & 0,022 & $\mathbf{0 , 0 1 7}$ & 0,292 & \\
\hline
\end{tabular}

Nota: $r$ - Coeficiente de correlação de Pearson. p-valor - Significância.

Legenda: CCAE - Custo Corrente / Aluno Equivalente; ATIPE - Aluno Tempo Integral / Professor Equivalente;

ATIFE - Aluno Tempo Integral / Funcionário Equivalente; FEPE - Funcionário Equivalente / Professor Equivalente; GPE - Grau de Participação Estudantil; GEDPG - Grau de Envolvimento Discente com Pós-Graduação; CCPG - Conceito CAPES/ MEC para a Pós-Graduação; IQCD - Índice de Qualificação do Corpo Docente; TSG - Taxa de Sucesso na Graduação.

Para realização da Análise de Agrupamentos e posteriormente da Análise Discriminante foi verificada a existência de valores extremos e de altas correlações (indícios de multicolinearidade), visando reduzir o risco de interferência e comprometimento da qualidade dos resultados da análise, conforme recomendado por Fávero et al (2009) e Hair et al (2009).

22

Pereira, A; Pereira, L; Tognini, M; Corassa, M; Carneiro, T - Classificação das Universidades Federais Brasileiras com base nos indicadores de gestão propostos pelo Tribunal de Contas da União - TCU 
A partir dos boxplot da Figura I, foram verificados 4 valores altamente discrepantes relatados pela UNIPAMPA (CCAE), UFV (FEPE), UNIFESP (GPE) e UNIFAP (CCPG), além dos casos da UTFPR que foram apontados como valores extremos em dois indicadores distintos (ATIFE e FEPE). Com base nessas constatações, essas universidades foram retiradas da análise de agrupamentos.

Os dados da Tabela 2, por sua vez, permitiram verificar a existência de dois casos de alta correlação entre os indicadores Conceito Capes/ MEC para a Pós-Graduação (CCPG) e Índice de Qualificação do Corpo Docente (IQCD) com o Grau de Envolvimento Discente com a PósGraduação (GEDPG). Dessa forma, optou-se pela retirada do indicador Grau de Envolvimento Discente com a Pós-Graduação da análise. Foram retiradas das análises ainda, as Universidades Federais da Fronteira Sul (UFFS), da Integração Latino-Americana (UNILA) e da Integração Internacional da Lusofonia Afro-Brasileira (UNILAB), em virtude da ausência de dados de diversos indicadores. Dessa forma, foram utilizados dados de 5I universidades e oito indicadores nas análises.

\subsection{Análise de Agrupamentos}

Para a análise de agrupamentos foi utilizado o método hierárquico aglomerativo com ligação de Ward para formação dos grupos. Para evitar possíveis efeitos das escalas dos indicadores, optou-se pela utilização de valores padronizados. O Dendograma gerado é apresentado na Figura 2. A partir da análise do Dendograma foi possível observar a formação de três grupos de instituições. A linha vertical no nível de distância Io permite visualizar a formação dos grupos. O Grupo I composto de 28 universidades, o Grupo 2 com I7 universidades e o Grupo 3 com 6 universidades. O Quadro I apresenta os membros de cada grupo formado.

\begin{tabular}{|l|l|}
\hline \multicolumn{1}{|c|}{ Quadro I - Membros dos grupos } \\
\hline \multirow{2}{|c|}{ Grupo } & \multicolumn{1}{c|}{ Universidades que compõem o grupo } \\
Grupo 1 - Maior porte & $\begin{array}{l}\text { FURG, UFAL, UFBA, UFC, UFCSPA, UFERSA, UFES, UFF, UFG, UFGD, UFJF, } \\
\text { UFLA, UFMG, UFMS, UFPA, UFPB, UFPE, UFPR, UFRGS, UFRJ, UFRN, UFSC, } \\
\text { UFSCAR, UFSM, UFT, UFU, UNIFAL-MG e UNIRIO }\end{array}$ \\
\hline Grupo 2 - Intermediárias & $\begin{array}{l}\text { UFABC, UFCG, UFMA, UFMT, UFOP, UFOPA, UFPEL, UFRA, UFRB, UFRPE, } \\
\text { UFRR, UFRRJ, UFSJ, UFTM, UFVJM, UNB e UNIFEI }\end{array}$ \\
\hline Grupo 3 - Menor porte & UFAC, UFAM, UFPI, UFS, UNIR e UNIVASF \\
\hline
\end{tabular}


A caracterização dos grupos foi feita a partir dos dados apresentados na Figura 3 e na Tabela 3. A Figura 3 apresenta dois gráficos do tipo radar que permitem comparar os valores de cada grupo para os indicadores de gestão e indicadores primários (usados para calcular os primeiros) mais o Índice Geral de Cursos de 2012 (IGC). A Tabela 3 apresenta os valores das estatísticas descritivas para cada grupo formado, complementando a Figura 3.

O Grupo I caracteriza-se por possuir os maiores valores em cinco indicadores, conforme verifica-se no Gráfico (a): Aluno Tempo Integral / Professor Equivalente (ATIPE), Grau de Participação Estudantil (GPE), Conceito Capes/MEC da Pós-Graduação (CCPG), Índice de Qualificação do Corpo Docente (IQCD) e Taxa de Sucesso da Graduação (TSG). O Gráfico (b) permite observar que o primeiro grupo formado possui os maiores valores em todos os indicadores primários analisados. Dessa forma, esse agrupamento pode ser descrito como grupo das maiores universidades federais brasileiras, tendo em vista que possuem os maiores números de alunos, professores e funcionários, além dos maiores valores de desempenho discente e custo corrente bruto.

O Grupo 2 possui os maiores valores dos indicadores Custo Corrente / Aluno Equivalente (CCAE) e Funcionário Equivalente / Professore Equivalente (FEPE), ao mesmo tempo que apresentou os menores valores de outros três indicadores - Aluno Tempo Integral / Professor Equivalente (ATIPE), Aluno tempo Integral / Funcionário Equivalente (ATIFE) e Grau de Participação Estudantil (GPE). Essa configuração aponta para uma quantidade pequena de alunos da graduação em relação ao tamanho da estrutura existente na universidade (representada pelo custo corrente, número de professores e funcionários). O Gráfico (b) da Figura 3 demonstra que esse grupo tem a menor média para o indicador primário Total de Alunos Regularmente Matriculados na Graduação (AG).

O Grupo 3 apresentou o maior valor do indicador Aluno Tempo Integral / Funcionário Equivalente (ATIFE) e os menores valores de cinco indicadores Custo Corrente / Aluno Equivalente (CCAE), Funcionário Equivalente / Professor Equivalente (FEPE), Conceito Capes/ MEC da Pós-Graduação (CCPG), Índice de Qualificação do Corpo Docente (IQCD) e Taxa de Sucesso da Graduação (TSG). O menor valor de Índice Geral de Cursos (IGC) apresentado no Gráfico (b), associado aos menores valores de CCPG e TSG, caracteriza o grupo como aquele de pior desempenho discente, além de possuir os menores níveis de qualificação dos professores e de investimento por aluno. 
Figura 2 - Dendograma

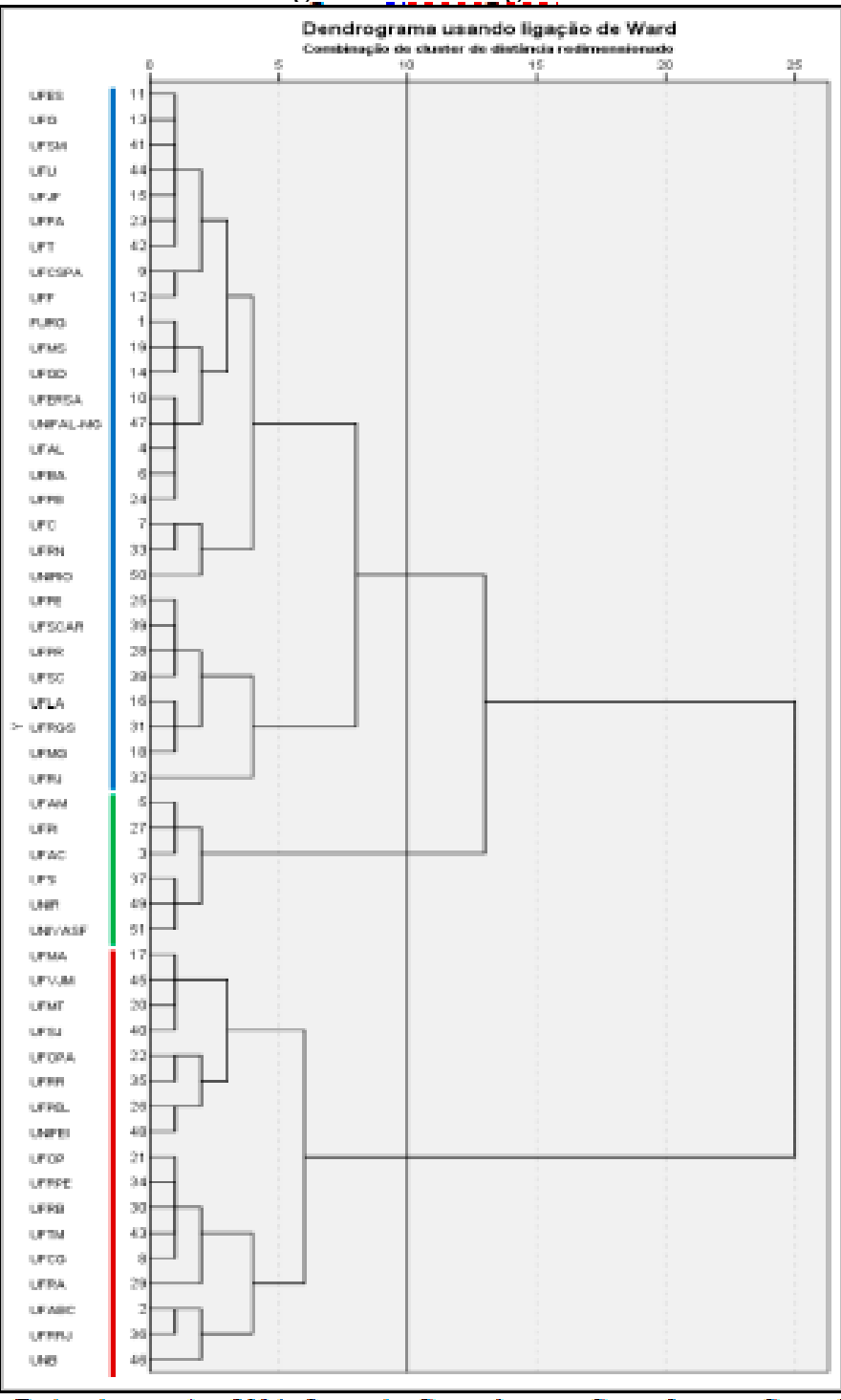

Fonte Dados da pesquisa, 2014. Legenda: Gropo l] Gropo $2=$ Grupo

Figura 3 - Gráfico Radar - Indicadores de Gestão e Primários

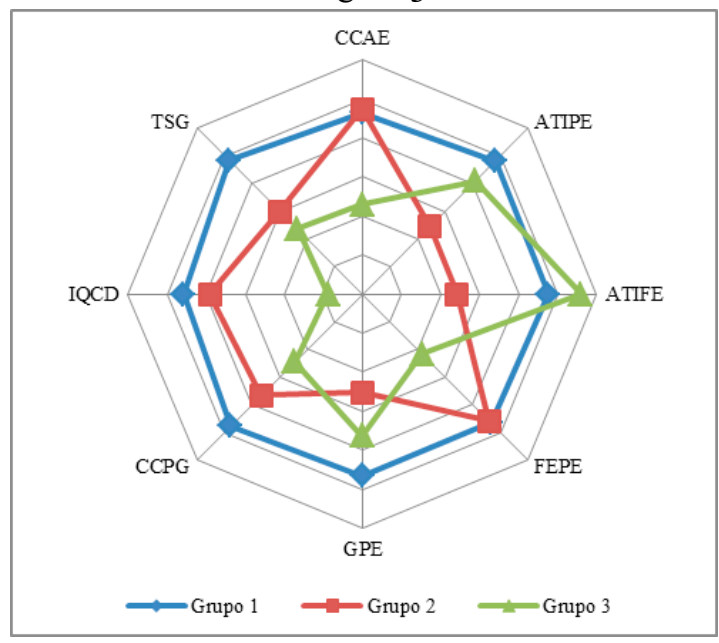

(a) Indicadores de Gestão

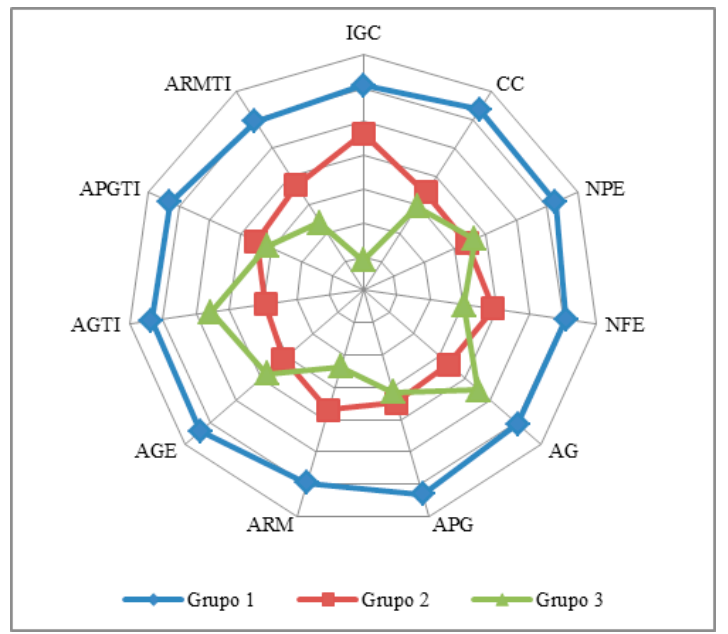

(b) Indicadores Primários 
Fonte: Dados da pesquisa, 20I4.

Legenda: CCAE - Custo Corrente / Aluno Equivalente, ATIPE - Aluno Tempo Integral / Professor Equivalente, ATIFE - Aluno tempo Integral / Funcionário Equivalente, FEPE - Funcionário Equivalente / Professore Equivalente, GPE - Grau de Participação Estudantil, Conceito Capes/MEC da Pós-Graduação (CCPG), IQCD - Índice de Qualificação do Corpo Docente, TSG - Taxa de Sucesso da Graduação, CC - Custo Corrente, NPE Número de Professores Equivalentes, NFE - Número de Funcionários Equivalentes, AG - Total de Alunos Regularmente Matriculados na Graduação, APG - Total de Alunos na Pós-graduação stricto sensu, ARM - Alunos de Residência Médica, AGE - Número de Alunos Equivalentes da Graduação, AGTI - Número de Alunos da Graduação em Tempo Integral, APGTI - Número de Alunos da Pós-graduação em Tempo Integral e ARTI - Número de Alunos de Residência Médica em Tempo Integral.

Tabela 3 - Estatísticas descritivas - Indicadores por Grupos

\begin{tabular}{|l|l|l|l|l|l|l|}
\hline \multirow{2}{*}{ Indicadores } & \multicolumn{2}{l|}{ Grupo 1 (n=28) } & \multicolumn{2}{l|}{ Grupo 2 (n=17) } & \multicolumn{2}{l|}{ Grupo 3 (n=6) } \\
\cline { 2 - 7 } & Média & $\begin{array}{l}\text { Desvio } \\
\text { padrão }\end{array}$ & Média & $\begin{array}{l}\text { Desvio } \\
\text { padrão }\end{array}$ & Média & $\begin{array}{l}\text { Desvio } \\
\text { padrão }\end{array}$ \\
\hline Custo Corrente / Aluno Equivalente (R\$) & $13.313,87$ & $2.060,99$ & $17.360,42$ & $3.188,70$ & $11.350,22$ & $1.746,49$ \\
\hline $\begin{array}{l}\text { Aluno Tempo Integral / Professor Equi- } \\
\text { valente }\end{array}$ & 13,12 & 1,77 & 8,85 & 1,78 & 11,26 & 2,47 \\
\hline $\begin{array}{l}\text { Aluno Tempo Integral / Funcionário } \\
\text { Equivalente }\end{array}$ & 9,22 & 1,78 & 5,72 & 1,46 & 10,24 & 1,22 \\
\hline $\begin{array}{l}\text { Funcionário Equivalente / Professor Equi- } \\
\text { valente }\end{array}$ & 1,46 & 0,27 & 1,62 & 0,40 & 1,11 & 0,27 \\
\hline Grau de Participação Estudantil & 0,78 & 0,11 & 0,56 & 0,10 & 0,65 & 0,12 \\
\hline $\begin{array}{l}\text { Conceito Capes/MEC para a Pós-Gradu- } \\
\text { ação }\end{array}$ & 3,99 & 0,53 & 3,58 & 0,44 & 3,19 & 0,15 \\
\hline Índice de Qualificação do Corpo Docente & 4,21 & 0,24 & 4,09 & 0,35 & 3,51 & 0,22 \\
\hline Taxa de Sucesso na Graduação & 63,79 & 16,43 & 45,52 & 13,77 & 39,66 & 14,59 \\
\hline
\end{tabular}

Fonte: Dados da pesquisa, 20I4.

Na sequência, a técnica de Análise Discriminante foi utilizada, conforme indicação de Fávero et al (2009), para confirmar a diferença entre os agrupamentos formados e apontar as variáveis que mais bem discriminam os grupos.

\subsection{Análise Discriminante}

A análise foi iniciada com os oito indicadores de gestão como variáveis independentes. O procedimento de seleção utilizado foi o Stepwise, associado à estatística Lambda de Wilks, considerando 5,0\% de significância para inclusão e I0,0\% para exclusão de variáveis independentes. Ao final de quatro etapas, foi confirmada a diferença entre os grupos e quatro indicadores de gestão (Aluno Tempo Integral / Funcionário Equivalente, Grau de Participação Estudantil, Custo Corrente / Aluno Equivalente e Índice de Qualificação do Corpo Docente) foram selecionados como variáveis que mais bem discriminam os agrupamentos.

A premissa de igualdade das matrizes de covariâncias da população foi testada com o teste Box’ M (23,50I; F Aprox.=0,9II; $p$-valor=0,573) que indicou não haver indícios de diferenças significativas entre as matrizes e confirmou a validade da análise realizada. Ademais, o valor da estatística Lambda de Wilks igual a 0,I50 associada a p-valor menor que 0,00I indicou que o modelo gerado possui baixa probabilidade de classificar incorretamente as universidades analisadas. 
A Tabela 4 apresenta a Matriz de Estrutura das funções discriminantes geradas. Com base nessa matriz verifica-se que os indicadores Aluno Tempo Integral / Funcionário Equivalente, Grau de Participação Estudantil, Custo Corrente / Aluno Equivalente são mais relevantes para a função discriminante I, enquanto o Índice de Qualificação do Corpo Docente é mais relevante para a função discriminante 2 .

Tabela 4: Matriz de Estrutura

\begin{tabular}{|l|c|c|}
\hline \multirow{2}{*}{ Indicadores } & \multicolumn{2}{c|}{ Função } \\
\cline { 2 - 3 } & 1 & $\mathbf{2}$ \\
\hline Aluno Tempo Integral / Funcionário Equivalente & 0,667 & $-0,311$ \\
\hline Grau de Participação Estudantil & 0,580 & 0,334 \\
\hline Custo Corrente / Aluno Equivalente & $-0,519$ & 0,351 \\
\hline Índice de Qualificação do Corpo Docente & 0,036 & 0,878 \\
\hline \multicolumn{2}{|c|}{ Fonte: Dados da pesquisa, 20I4. } \\
\hline
\end{tabular}

A Tabela 5 mostra os autovalores das funções e aponta que a função discriminante I explica $75,8 \%$ da variabilidade do modelo e a função discriminante 2 explica os $24,2 \%$ restantes.

Tabela 5: Autovalores das Funções Discriminantes

\begin{tabular}{|l|l|l|l|l|}
\hline $\begin{array}{l}\text { Função } \\
\text { Discriminante }\end{array}$ & Autovalores & $\%$ de variância & $\begin{array}{l}\text { \%o de variância } \\
\text { cumulativo }\end{array}$ & $\begin{array}{l}\text { Correlação } \\
\text { canônica }\end{array}$ \\
\hline 1 & 2,629 & 75,8 & 75,8 & 0,851 \\
\hline 2 & 0,840 & 24,2 & 100,0 & 0,676 \\
\hline
\end{tabular}

Fonte: Dados da pesquisa, 20I4.

Os valores de Lambda de Wilks e os testes de Qui-Quadrado para as funções são apresentados na Tabela 6. Os valores de Lambda de Wilks são consideravelmente diferentes de I,O e os p-valores dos testes são menores que 0,00I, o que significa que as duas funções possuem alto poder discriminante e são altamente significantes.

Tabela 6: Lambda de Wilks e Qui-quadrado para as funções

\begin{tabular}{|l|l|l|l|l|}
\hline Teste de funções & Lambda de Wilks & Qui-quadrado & gl & p-valor \\
\hline 1 até 2 & 0,150 & 88,290 & 8 & 0,000 \\
\hline 2 & 0,543 & 28,359 & 3 & 0,000 \\
\hline \multicolumn{4}{|c|}{ Fonte: Dados da pesquisa, 20I4. } \\
Nota: gl - Graus de liberdade. p-valor - Significância. \\
\hline
\end{tabular}

A Figura 4 mostra os centroides dos grupos baseados nas funções discriminantes I e 2. É possível perceber a distribuição das observações em torno dos centroides e verificar que as observações se concentram próximas de seu respectivo centroide. 
Figura 4: Centroides das funções discriminantes

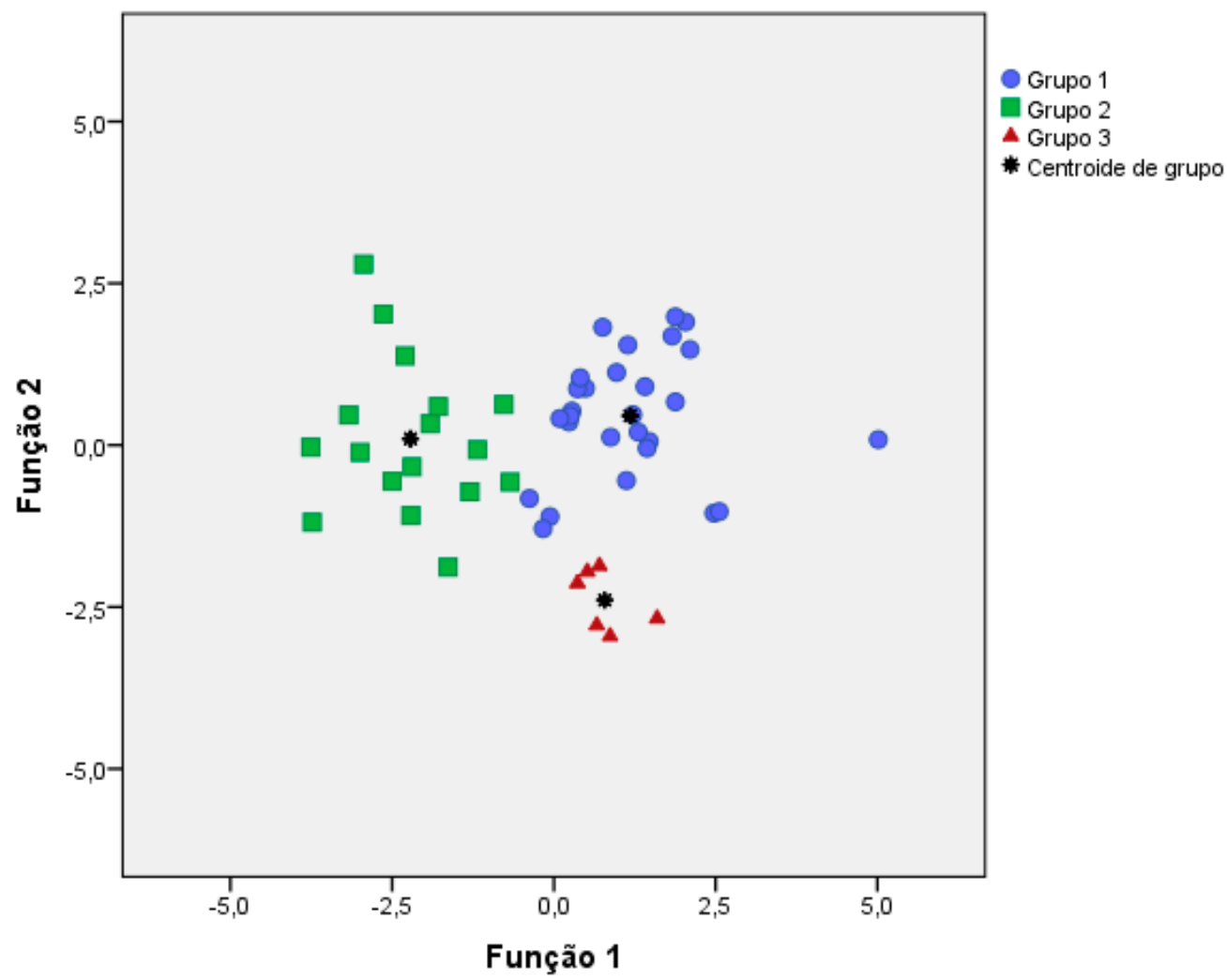

Fonte: Dados da pesquisa, 20I4.

O poder discriminante do modelo gerado foi avaliado por meio de uma Tabela de Classificação (Tabela 7) que mostrou I00\% de acerto na classificação das observações (dado original) nos grupos previstos com base nas funções discriminantes.

Tabela 7: Tabela de Classificação

\begin{tabular}{|c|c|c|c|c|c|c|}
\hline & \multicolumn{3}{|c|}{ Associação ao grupo prevista } & \multirow{2}{*}{ Total } \\
\hline & & & Grupo 1 & Grupo 2 & Grupo 3 & \\
\hline \multirow{6}{*}{$\begin{array}{c}\text { Original } \\
\text { (Observado) }\end{array}$} & \multirow{3}{*}{ Contagem } & Grupo 1 & 28 & 0 & 0 & 28 \\
\hline & & Grupo 2 & 0 & 17 & 0 & 17 \\
\hline & & Grupo 3 & 0 & 0 & 6 & 6 \\
\hline & \multirow{3}{*}{$\%$ de Acerto } & Grupo 1 & 100,0 & 0,0 & 0,0 & 100,0 \\
\hline & & Grupo 2 & 0,0 & 100,0 & 0,0 & 100,0 \\
\hline & & Grupo 3 & 0,0 & 0,0 & 100,0 & 100,0 \\
\hline
\end{tabular}

Com base nos resultados das análises de conglomerado e discriminante, foi possível confirmar a viabilidade de utilizar os indicadores de gestão propostos pelo TCU como classificadores das universidades federais. De acordo com os dados utilizados, em 20I2, os indicadores que mais bem discriminam as universidades analisadas foram Aluno em Tempo Integral / Funcionário Equivalente, Grau de Participação Estudantil, Custo Corrente / Aluno Equivalente 
que discriminou do grupo 2 - universidades de médio porte (maior custo por aluno, menos aluno por funcionário, menor grau de participação estudantil) dos grupos I - maiores universidades e 3 - menores universidades e o Índice de Qualificação do Corpo Docente que discriminou o grupo I - maiores (maior qualificação do corpo docente) do grupo 3 -menores (menor qualificação do corpo docente).

Pela análise comparada dos grupos por esses indicadores é possível concluir que as universidades de porte médio são menos eficientes na alocação de recursos financeiros e humanos quando comparadas as grandes e as pequenas. As grandes são mais eficientes por terem mais alunos proporcionalmente ao quadro de técnicos e ao orçamento para custeio além de terem mais alunos com dedicação integral aos cursos e melhor qualificação do corpo docente. As pequenas são mais eficientes na alocação de recursos do que as médias por terem menos funcionários e menos orçamento proporcionalmente ao número de alunos, embora apresentem os menores indicadores de qualidade de ensino: menor taxa de sucesso na graduação e menor conceito da Capes para os programas de pós-graduação.

No grupo 2 estão todas as universidades rurais. A maior parte das universidades desse grupo está localizada fora de capitais, no interior dos estados. No grupo 3 estão predominantemente universidades da região norte e nordeste.

Esses resultados indicam que as pequenas universidades são mais eficientes na alocação de recursos (por serem escassos) do que as médias, mas apresentam piores resultados acadêmicos. À medida que aumentam de porte, perdem eficiência na alocação de recursos, mas melhoram os resultados acadêmicos. Quando atingem portes maiores, mantem os bons resultados e recuperam a eficiência.

\section{CONSIDERAÇÕES FINAIS}

O objetivo deste trabalho foi classificar as universidades federais brasileiras em função dos indicadores de gestão propostos pelo TCU e, implicitamente, verificar o potencial desses indicadores como instrumentos para classificação das instituições federais de ensino superior. As instituições foram divididas em três grupos, com o auxílio da técnica de análise de agrupamentos, sendo o grupo I composto de 28 universidades, o grupo 2 de 17 universidades e o grupo 3 de seis IFES.

O Grupo I pode ser descrito como grupo das maiores universidades federais brasileiras, tendo em vista que possuem o maior número de alunos, professores e funcionários, além dos maiores valores para desempenho discente e custo corrente bruto. O Grupo 2 apresenta uma quantidade pequena de alunos da graduação em relação ao tamanho da estrutura existente na universidade (representada pelo custo corrente, número de professores e funcionários). O Grupo 3 caracteriza-se como de pior desempenho discente, além de possuir os menores níveis de qualificação dos professores e de investimento por aluno. 
O Grupo I reúne as universidades mais eficientes e com melhores indicadores de qualidade de ensino. No Grupo 2 estão as universidades menos eficientes na alocação de recursos e no Grupo 3 as universidades menos eficientes nos indicadores de qualidade do ensino.

O modelo discriminante gerado confirmou a diferença entre os grupos e foi capaz de classificar corretamente $100 \%$ das observações. Os indicadores Aluno Tempo Integral / Funcionário Equivalente, Grau de Participação Estudantil, Custo Corrente / Aluno Equivalente e Índice de Qualificação do Corpo Docente foram apontados como variáveis que mais bem discriminam os agrupamentos.

Por fim, foi confirmado o potencial dos indicadores de gestão para classificação das IFES e foi obtida uma classificação dessas instituições em grupos estatisticamente distintos, conforme estipulado nos objetivos do estudo. Para trabalhos futuros sugere-se avaliar a aplicação dessa metodologia em outras situações e reaplicar para outros períodos de tempo, verificando se a classificação se mantém estável ao longo do tempo e o grau de mobilidade entre os grupos.

\section{REFERÊNCIAS}

BRASIL. Lei n.Io.86I de I4 de abril de 2004. Institui o Sistema Nacional de Avaliação da Educação Superior - SINAES e dá outras providências. Diário Oficial da União, Brasília, DF, I5 de abril de 2004. Disponível em: <http://www.planalto.gov.br/>. Acesso em I5 ago 2014.

BRASIL. Tribunal de Contas da União - TCU; Secretaria de Educação Superior - SESu/MEC; Secretaria Federal de Controle Interno - SFC. Orientações para o cálculo dos indicadores de gestão: Decisão TCU n ${ }^{\circ}$ 408/2002 - plenário. Versão revisada em janeiro de 2009.

DIAS SOBRINHO, José. Avaliação: políticas educacionais e reformas da educação superior. São Paulo: Cortez, 2003.

FÁVERO, Luiz Paulo; BELFIORE, Patrícia; SILVA, Fabiana Lopes da; CHAN, Betty Lilian. Análise de dados: modelagem multivariada para tomada de decisões. Rio de Janeiro: Elsevier, 2009.

FREIRE, Fátima de Souza; CRISÓSTOMO, Vicente Lima; CASTRO, Juscelino Emanoel Gomes de. Análise do desempenho acadêmico e indicadores de gestão das IFES. Revista Produção Online, v. 7, n. 4, p. 5-25, 2008.

GUERRA, Herbert Newton Mota; BARBOSA, Francisco Vidal. As universidades federais em Minas Gerais: indicadores de desempenho e propostas de aprimoramento. ENCONTRO DA ASSOCIAÇÃO NACIONAL DE PÓS-GRADUAÇÃO EM ADMINISTRAÇÃO, 30, 2006, Salvador. Anais eletrônicos.... Disponível em: <http://www.anpad.org.br/enanpad/2006/dwn/ enanpad2006-apsb-2545.pdf>. Acesso em: 05 jul. 2014.

HAIR, Joseph F., Jr; BLACK, William C.; BABIN, Barry; ANDERSON, Rolph E.; TATHAN, Ronald L. Análise multivariada de dados. $6^{\mathrm{a}}$ ed. Porto Alegre: Bookman, 2009. 
MAROCO, João. Análise estatística: com a utilização do SPSS. Lisboa: Sílabo, 2003.

SANTOS, Clézia De Souza; CASTANEDA, Marcos Vinícius N. G.; BARBOSA, Jenny Dantas. Indicadores de desempenho das IFES da região nordeste: uma análise comparativa. 20II. XI Colóquio Internacional sobre Gestão Universitária na América do Sul. Disponível em: < https://repositorio.ufsc.br/handle/I23456789/25952 >. Acesso em: 05 jul. $20 \mathrm{I} 4$.

SILVA, César Augusto Tibúrcio; MORGAN, Beatriz Fátima; COSTA, Patrícia de Souza. Desenvolvimento e aplicação de uma metodologia para cálculo do custo-aluno de instituições públicas de ensino superior: um estudo de caso. Revista de Administração Pública, v. 38, n. 2, p. 243-260, 2004. 